\title{
Non-enzymatic Acylative Kinetic Resolution of Baylis-Hillman Adducts
}

Ciarán Ó Dálaigh and Stephen J. Connon *

Table of Contents

$\begin{array}{lll}1.0 & \text { General } & \text { S2 }\end{array}$

2.0 Synthesis of catalysts 1a-1e and the synthesis-resolution of 9a-10a S3

3.0 Spectral data for uncharacterized esters $\quad$ S13

4.0 HPLC conditions/retention times and optical rotation data S15

$\begin{array}{llr}\text { 5.0 NMR Spectra } & \text { S20 }\end{array}$

$\begin{array}{llr}\text { 6.0 } & \text { References } & \text { S26 }\end{array}$ 


\subsection{General}

Proton Nuclear Magnetic Resonance spectra were recorded on a $400 \mathrm{MHz}$ spectrometer in $\mathrm{CDCl}_{3}$ referenced relative to residual $\mathrm{CHCl}_{3}(\delta=7.26 \mathrm{ppm})$. Chemical shifts are reported in ppm and coupling constants in Hertz. Carbon NMR spectra were recorded on the same instrument (100 MHz) with total proton decoupling. All melting points are uncorrected. Flash chromatography was carried out using silica gel, particle size 0.04-0.063 mm. TLC analysis was performed on precoated $60 \mathrm{~F}_{254}$ slides, and visualised by either $\mathrm{UV}$ irradiation or $\mathrm{KMnO}_{4}$ staining. Optical rotation measurements are quoted in units of $10^{-1} \mathrm{deg} \mathrm{cm}^{2} \mathrm{~g}^{-1}$. Toluene, ether and THF were distilled from sodium. Methylene chloride and triethylamine were distilled from calcium hydride. Analytical CSP-HPLC was performed using CHIRALCEL OD-H (4.6 mm x 25 $\mathrm{cm})$ and CHIRALCEL AS-H (4.6 mm x $25 \mathrm{~cm})$ columns. Unless otherwise stated, all chemicals were obtained from commercial sources and used as received. $m$-terphenylmagnesium bromide, ${ }^{1}$ 3,5-dimethoxyphenylmagnesium bromide, ${ }^{2}$ 3,5-bis(trifluromethyl)phenylmagnesium bromide, ${ }^{3}$ were synthesized according to literature procedures. Unless otherwise specified, all reactions were carried out in oven-dried glassware with magnetic stirrers under an atmosphere of argon. 


\subsection{Synthesis of catalysts 1a-1e}

\section{General outline}

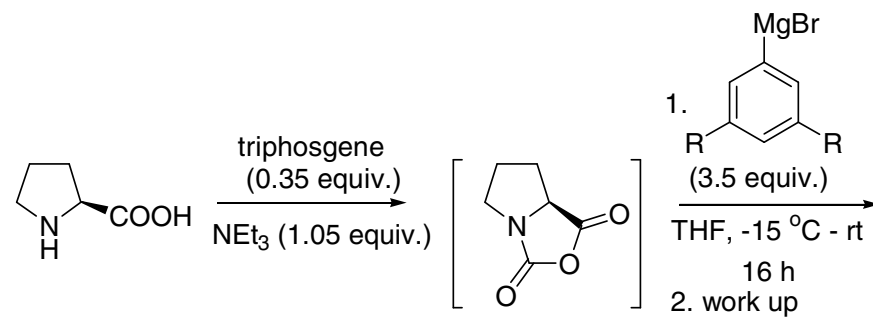

A $\mathrm{Ar}=3,5-\left(\mathrm{C}_{6} \mathrm{H}_{5}\right)_{2}-\mathrm{C}_{6} \mathrm{H}_{3}$ B $\mathrm{Ar}=3,5-\left(\mathrm{OCH}_{3}\right)_{2}-\mathrm{C}_{6} \mathrm{H}_{3}$ C $\mathrm{Ar}=3,5-\left(\mathrm{CH}_{3}\right)_{2}-\mathrm{C}_{6} \mathrm{H}_{3}$ D $\mathrm{Ar}=3,5-\left(\mathrm{CF}_{3}\right)_{2}-\mathrm{C}_{6} \mathrm{H}_{3}$

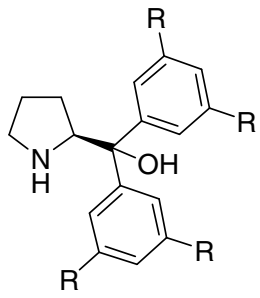

E $\mathrm{Ar}=3,5-\left(\mathrm{C}_{6} \mathrm{H}_{5}\right)_{2}-\mathrm{C}_{6} \mathrm{H}_{3} \quad 57 \%$ F $\mathrm{Ar}=3,5-\left(\mathrm{OCH}_{3}\right)_{2}-\mathrm{C}_{6} \mathrm{H}_{3} 17 \%$ G $\mathrm{Ar}=3,5-\left(\mathrm{CH}_{3}\right)_{2}-\mathrm{C}_{6} \mathrm{H}_{3} \quad 54 \%$ H $\mathrm{Ar}=3,5-\left(\mathrm{CF}_{3}\right)_{2}-\mathrm{C}_{6} \mathrm{H}_{3} \quad 54 \%$

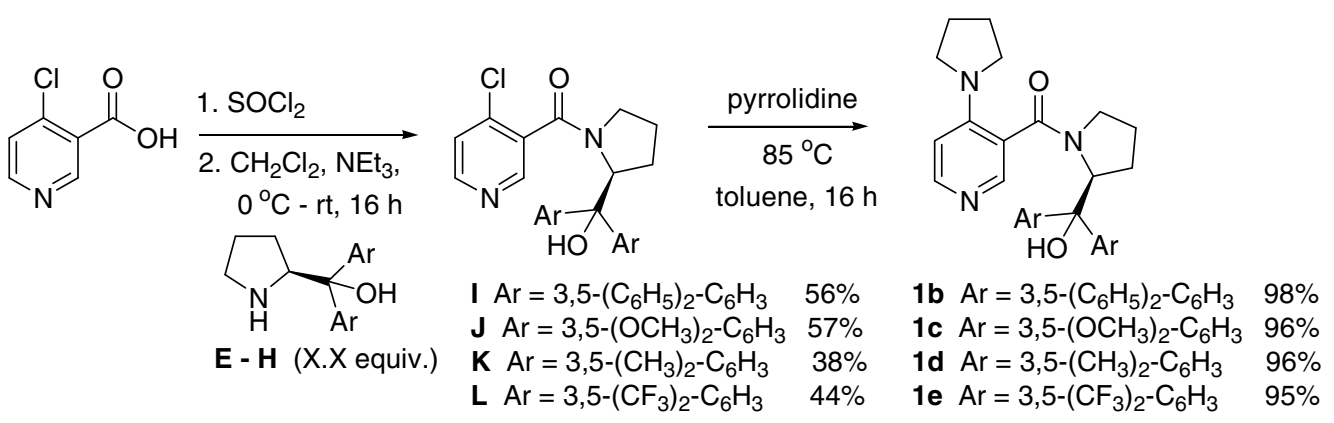

\section{Prolinol E}

A $25 \mathrm{~mL}$ three-necked round bottom flask fitted with an Ar inlet tube, $10 \mathrm{~mL}$ addition funnel and a Teflon-coated thermocouple probe, containing dry THF $(5 \mathrm{~mL})$ was charged with $(S)$-proline (187 mg, $1.627 \mathrm{mmol})$. To the well-stirred, cooled $\left(15-20^{\circ} \mathrm{C}\right)$ suspension was added a solution of tri-phosgene $(160 \mathrm{mg}, 0.542 \mathrm{mmol})$ in $\mathrm{THF}(5 \mathrm{~mL})$ over a $30 \mathrm{~min}$ period, maintaining the internal temperature at $15-20{ }^{\circ} \mathrm{C}$. The mixture was warmed to $30-40{ }^{\circ} \mathrm{C}$ and aged for $1 \mathrm{~h}$. Once homogeneous, the reaction mixture was allowed stir for an additional $0.5 \mathrm{~h}$ at $30-35{ }^{\circ} \mathrm{C}$ and then cooled to $15-20{ }^{\circ} \mathrm{C}$. While maintaining the internal temperature at $15-20{ }^{\circ} \mathrm{C}$, the reaction mixture was concentrated in vacuo to a volume of $2 \mathrm{~mL}$. The residue was dissolved in dry THF (8 $\mathrm{mL})$ and the solution was cooled to $0-5{ }^{\circ} \mathrm{C}$. With good agitation, $\operatorname{dry} \mathrm{NEt}_{3}(237 \mu \mathrm{L}, 1.71 \mathrm{mmol})$ was added over 15 min while maintaining the internal temperature at $0-5{ }^{\circ} \mathrm{C}$. After the addition was 
complete, the mixture was aged for $30 \mathrm{~min}$ at $0{ }^{\circ} \mathrm{C}$ and then filtered through a medium frit, sintered-glass funnel. The resultant cake of $\mathrm{NEt}_{3} . \mathrm{HCl}$ was washed with $\mathrm{THF}(5 \mathrm{~mL} \times 2)$. The filtrate and THF washes were combined to afford a solution containing $(S)$-pyrrolo-oxazole product that was used immediately without further purification.

A $50 \mathrm{~mL}$ three-necked flask fitted with a $25 \mathrm{~mL}$ addition funnel containing the THF solution of the pyrrolo-oxazole $(1.6 \mathrm{mmol}$ in $20 \mathrm{~mL})$ and a stirrer was charged with a solution of $\mathrm{m}$ terphenyl magnesium bromide $(5.7 \mathrm{mmol})$ in THF $(10 \mathrm{~mL})$ and cooled to $-15{ }^{\circ} \mathrm{C}$. The THF solution of pyrrolo-oxazole was added over a $30 \mathrm{~min}$ period while maintaining the internal temperature at -10 to $-15{ }^{\circ} \mathrm{C}$. After the addition was complete, the mixture was allowed warm to room temperature and aged for $16 \mathrm{~h}$. The reaction was quenched with the addition of $2 \mathrm{M}$ aqueous $\mathrm{H}_{2} \mathrm{SO}_{4}(2 \mathrm{~mL})$, over a $0.5 \mathrm{~h}$ period, while maintaining the internal temperature below 20 ${ }^{\circ} \mathrm{C}$. During the quench a thick white precipitate of $\mathrm{MgSO}_{4}$ formed. The mixture was agitated for $1 \mathrm{~h}$ at $0{ }^{\circ} \mathrm{C}$ and filtered through a medium-frit, sintered-glass funnel. The $\mathrm{MgSO}_{4}$ cake was washed free of residual product with THF $(3 \times 10 \mathrm{~mL})$. The filtrate and THF washes were combined and concentrated at atmospheric pressure to $10 \%$ of the original volume. The product as its sulfate salt failed to precipitate from solution. The crude mixture was basified with $2 \mathrm{M}$ $\mathrm{NaOH}(4 \mathrm{~mL}, 8 \mathrm{mmol})$ and diluted with $\mathrm{CH}_{2} \mathrm{Cl}_{2}(25 \mathrm{~mL})$. The organic layer was washed with $\mathrm{H}_{2} \mathrm{O}\left(2 \times 25 \mathrm{~mL}\right.$ ), dried with $\mathrm{MgSO}_{4}$ and concentrated in vacuo. Purification by column chromatography (99:1 $\mathrm{CH}_{2} \mathrm{Cl}_{2}-\mathrm{NEt}_{3}$ ) gave prolinol E (512 mg, $57 \%$ ) as a white solid, m.p.: 92$94{ }^{\circ} \mathrm{C} .[\alpha]_{\mathrm{D}}{ }^{20}=-62.2\left(\mathrm{c} 0.143, \mathrm{CHCl}_{3}\right) .{ }^{1} \mathrm{H} \mathrm{NMR}\left(\mathrm{CDCl}_{3}\right) \delta 7.90(\mathrm{~s}, 2 \mathrm{H}), 7.80(\mathrm{~s}, 2 \mathrm{H}), 7.74-7.60$ (m, 10H), 7.49-7.41 (m, 8H), 7.40-7.36 (m, 4H), $4.56(\mathrm{~m}, 1 \mathrm{H}), 2.96(\mathrm{~m}, 2 \mathrm{H}), 1.92-1.70(\mathrm{~m}, 4 \mathrm{H})$. ${ }^{13} \mathrm{C} \mathrm{NMR}\left(\mathrm{CDCl}_{3}\right) \delta 145.9,144.3,141.8,141.4,141.2,141.0,128.6,128.5,128.4,128.3,127.3$, 
127.2, 124.7, 124.4, 123.7, 123.3, 77.4, 64.7, 46.6, 26.5, 25.2. IR (film) 3356, 2968, 2869, 2299, 1594, 1428, $1264 \mathrm{~cm}^{-1}$. HRMS (ESI, m/z) calcd for $\mathrm{C}_{41} \mathrm{H}_{36} \mathrm{NO}(\mathrm{M}+\mathrm{H})^{+}$558.2797, found 558.2791.

\section{Prolinol F}

Prepared as per the synthesis of prolinol E using (S)-pyrrolo-oxazole $(2.88 \mathrm{mmol}$ in $20 \mathrm{~mL})$ and 3,5-dimethoxyphenylmagnesium bromide $(8.64 \mathrm{mmol})$ in THF $(40 \mathrm{~mL})$. Purification by column chromatography (99:1, EtOAc-NEt $\left.3, \mathrm{R}_{\mathrm{f}} 0.2\right)$ gave prolinol $\mathbf{F}(185 \mathrm{mg}, 17 \%)$ as a clear oil. $[\alpha]_{\mathrm{D}}{ }^{20}$ $=-81\left(\mathrm{c} 0.12, \mathrm{CHCl}_{3}\right) .{ }^{1} \mathrm{H} \mathrm{NMR}\left(\mathrm{CDCl}_{3}\right) \delta 6.77(\mathrm{~s}, 2 \mathrm{H}), 6.69(\mathrm{~s}, 2 \mathrm{H}), 6.30(\mathrm{~s}, 2 \mathrm{H}), 4.19(\mathrm{dd}, 1 \mathrm{H}$, $\mathrm{J}=7.5,7.5 \mathrm{~Hz}), 3.79(\mathrm{~s}, 6 \mathrm{H}), 3.78(\mathrm{~s}, 6 \mathrm{H}), 2.98(\mathrm{~m}, 2 \mathrm{H}), 1.78-1.57(\mathrm{~m}, 4 \mathrm{H}) .{ }^{13} \mathrm{C} \mathrm{NMR}\left(\mathrm{CDCl}_{3}\right) \delta$ 160.2, 159.9, 103.8, 103.3, 98.0, 97.8, 76.8, 64.2, 54.9, 54.8, 46.2, 25.8, 24.8. IR (film) 3344, 2936, 2837, 1592, 1425, 1203, $1152 \mathrm{~cm}^{-1}$. HRMS (ESI, m/z) calcd for $\mathrm{C}_{21} \mathrm{H}_{27} \mathrm{NO}_{5} \mathrm{Na}(\mathrm{M}+\mathrm{Na})^{+}$ 396.1923, found 396.1927.

\section{Prolinol G}

Prepared as per the synthesis of prolinol E using $(S)$-pyrrolo-oxazole $(6.52 \mathrm{mmol}$ in $20 \mathrm{~mL})$ and (3,5-dimethylphenyl)magnesium bromide $(19.55 \mathrm{mmol})$ in THF $(40 \mathrm{~mL})$. Purification by column chromatography (98:2, $\left.\mathrm{CH}_{2} \mathrm{Cl}_{2}-\mathrm{NEt}_{3} \mathrm{R}_{\mathrm{f}} 0.3\right)$ gave prolinol $\mathbf{G}(1.100 \mathrm{~g}, 54 \%)$ as a white solid, m.p. 96-98 ${ }^{\circ} \mathrm{C}\left(\right.$ lit. $\left.^{4} 97.5-98{ }^{\circ} \mathrm{C}\right) .{ }^{1} \mathrm{H}$ NMR $\left(\mathrm{CDCl}_{3}\right) \delta 7.19$ (s, 2H), 7.12 (s, 2H), 6.81 (s, 2H), 4.24 $(\mathrm{dd}, 1 \mathrm{H}, \mathrm{J}=8.0,7.5 \mathrm{~Hz}), 3.08-2.92(\mathrm{~m}, 2 \mathrm{H}), 2.30(\mathrm{~s}, 6 \mathrm{H}), 2.29(\mathrm{~s}, 6 \mathrm{H}), 1.78-1.55(\mathrm{~m}, 4 \mathrm{H})$. 


\section{Prolinol H}

Prepared as per the synthesis of prolinol E using $(S)$-pyrrolo-oxazole (8.68 mmol in $20 \mathrm{~mL})$ and 3,5-[bis(trifluromethyl)phenyl]magnesium bromide $(33 \mathrm{mmol})$ in THF (30 mL). Purification by column chromatography (99:1 EtOAc- $\left.\mathrm{NEt}_{3}, \mathrm{R}_{\mathrm{f}} 0.3\right)$ gave prolinol $\mathbf{H}(2.45 \mathrm{~g}, 54 \%)$ as a white solid, m.p. 102-104 ${ }^{\circ} \mathrm{C} .{ }^{1} \mathrm{H}$ NMR $\left(\mathrm{CDCl}_{3}\right) \delta 8.06$ (s, 2H), 7.98 (s, 2H), 7.79 (s, 2H), 4.37 (dd, 1H, $\mathrm{J}=7.5,7.5 \mathrm{~Hz}), 3.09(\mathrm{~m}, 2 \mathrm{H}), 1.86-1.77(\mathrm{~m}, 2 \mathrm{H}), 1.72(\mathrm{~m}, 1 \mathrm{H}, \mathrm{NH}), 1.61-1.51(\mathrm{~m}, 2 \mathrm{H})$. Spectral data for this compound is consistent with that in the literature. ${ }^{5}$

\section{Chloropyridine I}

A $10 \mathrm{~mL}$ round bottom flask charged with 4-chloronicotinic acid $(68 \mathrm{mg}, 0.431 \mathrm{mmol})$ and $\mathrm{SOCl}_{2}(1.0 \mathrm{~mL})$ was fitted with a reflux condenser and heated at $90{ }^{\circ} \mathrm{C}$ for 1 hour. Removal of $\mathrm{SOCl}_{2}$ by distillation gave 4-chloronicotinic acid chloride hydrochloride as a yellow solid, which was placed under an atmosphere of $\mathrm{Ar}$, cooled to $0{ }^{\circ} \mathrm{C}$ and suspended in $\mathrm{CH}_{2} \mathrm{Cl}_{2}(3 \mathrm{~mL})$ added via syringe. Subsequently a solution of prolinol E $(200 \mathrm{mg}, 0.359 \mathrm{mmol})$ and $\mathrm{NEt}_{3}(200 \mu \mathrm{L}$, $1.44 \mathrm{mmol})$ in $\mathrm{CH}_{2} \mathrm{Cl}_{2}(5 \mathrm{~mL})$, was added via syringe. The yellow solution was left to stir overnight. $\mathrm{CH}_{2} \mathrm{Cl}_{2}(30 \mathrm{~mL})$ was then added and the resulting solution washed with $\mathrm{NaHCO}_{3}(2 \mathrm{x}$ $40 \mathrm{~mL})$, and brine $(2 \times 40 \mathrm{~mL})$. The organic extracts were separated, dried $\left(\mathrm{MgSO}_{4}\right)$ and the solvent removed in vacuo. Purification by column chromatography $\left(9: 1 \mathrm{CH}_{2} \mathrm{Cl}_{2}\right.$-EtOAc, $\left.\mathrm{R}_{\mathrm{f}} 0.3\right)$ gave chloropyridine I $(141 \mathrm{mg}, 56 \%)$ as a white solid, m.p. $136-137{ }^{\circ} \mathrm{C} \cdot[\alpha]_{\mathrm{D}}{ }^{20}=-117.5(\mathrm{c}$ 0.143, $\left.\mathrm{CHCl}_{3}\right) .{ }^{1} \mathrm{H} \mathrm{NMR}\left(\mathrm{CDCl}_{3}\right) \delta 8.50(\mathrm{bs}, 1 \mathrm{H}), 7.92(\mathrm{~s}, 2 \mathrm{H}), 7.85-7.80(\mathrm{~m}, 4 \mathrm{H}), 7.67(\mathrm{~m}, 8 \mathrm{H})$, 7.49-7.45 (m, 9H), 7.41-7.30 (m, 5H), $6.72(\mathrm{~s}, 1 \mathrm{H}, \mathrm{OH}), 5.57(\mathrm{dd}, 1 \mathrm{H}, \mathrm{J}=7.0,7.0 \mathrm{~Hz}), 3.12-2.91$ $(\mathrm{m}, 2 \mathrm{H}), 2.40-2.28(\mathrm{~m}, 2 \mathrm{H}), 1.78-1.62(\mathrm{~m}, 1 \mathrm{H}), 1.59-1.38(\mathrm{~m}, 1 \mathrm{H}) .{ }^{13} \mathrm{C} \mathrm{NMR}\left(\mathrm{CDCl}_{3}\right) \delta 167.5$, $150.5,150.4,147.8,146.2,143.8,141.6,141.4,141.2,141.0,129.0,128.9,128.8,127.6,127.5$ 
127.4, 127.3, 127.2, 125.8, 125.7, 125.6, 125.5, 82.2, 68.4, 50.4, 30.4, 23.9. IR (film) 3275, 2962, 2245, 1575, 1427, 1185, $1030 \mathrm{~cm}^{-1}$. HRMS (ESI, m/z) calcd for $\mathrm{C}_{47} \mathrm{H}_{38} \mathrm{~N}_{2} \mathrm{O}_{2} \mathrm{Cl}(\mathrm{M}+\mathrm{H})^{+}$ 697.2622, found 697.2640

\section{Chloropyridine J}

Prepared as per the synthesis of chloropyridine I using 4-chloronicotinic acid (86 mg, 0.547 mmol), $\mathrm{SOCl}_{2}(1.0 \mathrm{~mL}), \mathrm{CH}_{2} \mathrm{Cl}_{2}(3+5 \mathrm{~mL})$, prolinol $\mathbf{F}(170 \mathrm{mg}, 0.456 \mathrm{mmol})$ and $\mathrm{NEt}_{3}(253$ $\mu \mathrm{L}, 1.82 \mathrm{mmol})$. Purification by column chromatography $\left(1: 1 \mathrm{CHCl}_{2}-\mathrm{EtOAc}, \mathrm{R}_{\mathrm{f}} 0.3\right)$ gave chloropyridine $\mathbf{J}(132 \mathrm{mg}, 57 \%)$ as a white solid, m.p. $70-72{ }^{\circ} \mathrm{C} .[\alpha]_{\mathrm{D}}{ }^{20}=-98\left(\mathrm{c} 0.126, \mathrm{CHCl}_{3}\right)$.

${ }^{1} \mathrm{H}$ NMR $\left(\mathrm{CDCl}_{3}\right) \delta 8.53(\mathrm{~d}, 1 \mathrm{H}, \mathrm{J}=5.5 \mathrm{~Hz}), 8.30-8.15(\mathrm{bs}, 1 \mathrm{H}), 7.37(\mathrm{~d}, 1 \mathrm{H}, \mathrm{J}=5.5 \mathrm{~Hz}), 6.78(\mathrm{~s}$, 2H), $6.67(\mathrm{~s}, 2 \mathrm{H}), 6.61(\mathrm{~s}, 1 \mathrm{H}), 6.46(\mathrm{~s}, 1 \mathrm{H}), 6.41(\mathrm{~s}, 1 \mathrm{H}), 5.19(\mathrm{dd}, 1 \mathrm{H}, \mathrm{J}=7.5,8.0 \mathrm{~Hz}), 3.80(\mathrm{~s}$, $6 \mathrm{H}), 3.79(\mathrm{~s}, 6 \mathrm{H}), 3.17(\mathrm{~m}, 2 \mathrm{H}), 2.14(\mathrm{~m}, 2 \mathrm{H}), 1.73-1.48(\mathrm{~m}, 2 \mathrm{H}) .{ }^{13} \mathrm{C} \mathrm{NMR}\left(\mathrm{CDCl}_{3}\right) \delta: 166.4$, 159.9, 159.8, 149.7, 147.0, 146.8, 144.6, 139.1, 131.4, 124.5, 105.9, 105.7, 98.7, 98.6, 81.2, 68.4, 55.0, 54.9, 49.2, 29.9, 23.5. IR (film): 3275, 2938, 2837, 1594, 1425, 1203, $1060 \mathrm{~cm}^{-1}$. HRMS (ESI, m/z) calcd for $\mathrm{C}_{27} \mathrm{H}_{30} \mathrm{ClN}_{2} \mathrm{O}_{6}(\mathrm{M}+\mathrm{H})^{+} 513.1792$, found 513.1805

\section{Chloropyridine K}

Prepared as per the synthesis of chloropyridine I using 4-chloronicotinic acid (440 mg, 2.79 mmol), $\mathrm{SOCl}_{2}(2.0 \mathrm{~mL}), \mathrm{CH}_{2} \mathrm{Cl}_{2}(6+10 \mathrm{~mL})$, prolinol $\mathbf{G}(720 \mathrm{mg}, 2.32 \mathrm{mmol})$ and $\mathrm{NEt}_{3}(1.29$ $\mathrm{mL}, 9.28 \mathrm{mmol})$. Purification by column chromatography $\left(1: 1 \mathrm{CH}_{2} \mathrm{Cl}_{2}\right.$-EtOAc, $\left.\mathrm{R}_{\mathrm{f}} 0.4\right)$ gave chloropyridine $\mathbf{K}(394 \mathrm{mg}, 38 \%)$ as a white solid, m.p. $73-75^{\circ} \mathrm{C} .[\alpha]_{\mathrm{D}}{ }^{20}=-117.5$ (c 0.143 , $\left.\mathrm{CHCl}_{3}\right) .{ }^{1} \mathrm{H}$ NMR $\left(\mathrm{CDCl}_{3}\right) \delta 8.52(\mathrm{~d}, 1 \mathrm{H}, \mathrm{J}=5.5 \mathrm{~Hz}), 8.30-8.10(\mathrm{bs}, 1 \mathrm{H}), 7.37(\mathrm{~d}, 1 \mathrm{H}, \mathrm{J}=5.5$ Hz), $7.21(\mathrm{~s}, 2 \mathrm{H}), 7.08(\mathrm{~s}, 2 \mathrm{H}), 6.99(\mathrm{~s}, 1 \mathrm{H}), 6.95(\mathrm{~s}, 1 \mathrm{H}), 6.20(\mathrm{~s}, 1 \mathrm{H}), 5.35(\mathrm{dd}, 1 \mathrm{H}, \mathrm{J}=6.5,6.5$ 
Hz), $3.08(\mathrm{~m}, 1 \mathrm{H}), 2.85(\mathrm{~m}, 1 \mathrm{H}), 2.35(\mathrm{~s}, 6 \mathrm{H}), 2.32(\mathrm{~s}, 6 \mathrm{H}), 2.25(\mathrm{~m}, 1 \mathrm{H}), 2.11(\mathrm{~m}, 1 \mathrm{H}), 1.60(\mathrm{~m}$, 1H), $1.34(\mathrm{~m}, 1 \mathrm{H}) .{ }^{13} \mathrm{C}$ NMR $\left(\mathrm{CDCl}_{3}\right) \delta 166.5,150.5,147.7,144.9,142.4,139.8,139.7,136.9$, 136.6, 132.5, 128.6, 125.3, 125.0, 124.2, 81.4, 67.3, 49.9, 29.5, 23.4, 21.2, 21.1. IR (film) 3303, 2917, 2245, 1619, 1576, 1435, 1156. HRMS (ESI, m/z) calcd for $\mathrm{C}_{27} \mathrm{H}_{29} \mathrm{~N}_{2} \mathrm{O}_{2} \mathrm{NaCl}(\mathrm{M}+\mathrm{Na})^{+}$ 471.1815, found 471.1824 .

\section{Chloropyridine L}

Prepared as per the synthesis of chloropyridine I using 4-chloronicotinic acid (144 mg, 0.914 mmol), $\mathrm{SOCl}_{2}(1.0 \mathrm{~mL}), \mathrm{CH}_{2} \mathrm{Cl}_{2}(3+5 \mathrm{~mL})$, prolinol $\mathbf{H}(400 \mathrm{mg}, 0.762 \mathrm{mmol})$ and $\mathrm{NEt}_{3}(540$ $\mu \mathrm{L}, 3.90 \mathrm{mmol})$. Purification by column chromatography $\left(1: 1 \mathrm{CH}_{2} \mathrm{Cl}_{2}-\mathrm{EtOAc}\right.$, Rf 0.3) gave chloropyridine $\mathbf{L}(222 \mathrm{mg}, 44 \%)$ as a white solid, m.p. $78-80{ }^{\circ} \mathrm{C} .[\alpha]_{\mathrm{D}}{ }^{20}=-67.5$ (c 0.157 , $\left.\mathrm{CHCl}_{3}\right) .{ }^{1} \mathrm{H} \mathrm{NMR}\left(\mathrm{CDCl}_{3}\right) \delta 8.55(\mathrm{~d}, 1 \mathrm{H}, \mathrm{J}=5.5 \mathrm{~Hz}), 8.40-8.22(\mathrm{bs}, 1 \mathrm{H}), 8.08(\mathrm{~s}, 2 \mathrm{H}), 7.96(\mathrm{~s}$, 1H), $7.89(\mathrm{~s}, 3 \mathrm{H}), 7.37(\mathrm{~d}, 1 \mathrm{H}, \mathrm{J}=5.5 \mathrm{~Hz}), 7.17(\mathrm{~s}, 1 \mathrm{H}), 5.31(\mathrm{dd}, 1 \mathrm{H}, \mathrm{J}=8.0,8.0 \mathrm{~Hz}), 3.28(\mathrm{~m}$, $1 \mathrm{H}), 3.08(\mathrm{~m}, 1 \mathrm{H}), 2.20(\mathrm{~m}, 1 \mathrm{H}), 1.97(\mathrm{~m}, 1 \mathrm{H}), 1.76(\mathrm{~m}, 1 \mathrm{H}), 1.50(\mathrm{~m}, 1 \mathrm{H}) .{ }^{13} \mathrm{C} \mathrm{NMR}\left(\mathrm{CDCl}_{3}\right) \delta$ 167.9, 151.1, 147.4, 146.0, 144.2, 139.6, 131.4, $131.3(\mathrm{~J}=34.0 \mathrm{~Hz}), 131.2(\mathrm{~J}=34.0 \mathrm{~Hz}), 127.4$, 127.0, 124.2, $122.7(\mathrm{~J}=271.4 \mathrm{~Hz}), 122.6(\mathrm{~J}=271.4 \mathrm{~Hz}), 121.9,121.8,80.1,67.7,50.0,29.7$, 23.5. IR (film) 3368, 2923, 2853, 1623, 1462, 1277, $1130 \mathrm{~cm}^{-1}$. HRMS (ESI, m/z) calcd for $\mathrm{C}_{27} \mathrm{H}_{18} \mathrm{~N}_{2} \mathrm{O}_{2} \mathrm{~F}_{12} \mathrm{Cl}(\mathrm{M}+\mathrm{H})^{+}$665.0865, found 665.0881.

\section{Catalyst 1b}

A $10 \mathrm{~mL}$ round bottom flask was charged with chloropyridine $\mathbf{I}(121 \mathrm{mg}, 0.17 \mathrm{mmol})$ and toluene $(2 \mathrm{~mL})$ with stirring. To this was added pyrrolidine $(145 \mu \mathrm{L}, 1.7 \mathrm{mmol})$ via syringe. The flask was fitted with a reflux condenser and heated at $85^{\circ} \mathrm{C}$ for $16 \mathrm{~h} . \mathrm{CH}_{2} \mathrm{Cl}_{2}(20 \mathrm{~mL})$ was then 
added and the solution washed with $\mathrm{NaHCO}_{3}(2 \times 30 \mathrm{~mL})$ and brine $(2 \times 30 \mathrm{~mL})$. The organic extracts were separated, dried $\left(\mathrm{MgSO}_{4}\right)$ and the solvent removed in vacuo. Purification by column chromatography $\left(1: 1 \mathrm{Hex}-\mathrm{CH}_{2} \mathrm{Cl}_{2}, \mathrm{R}_{\mathrm{f}} 0.2\right)$ gave catalyst $\mathbf{1 b}(124 \mathrm{mg}, 98 \%)$ as a white solid, m.p. $253-254{ }^{\circ} \mathrm{C} .[\alpha]_{\mathrm{D}}{ }^{20}=-37\left(\mathrm{c} 0.1, \mathrm{CHCl}_{3}\right) .{ }^{1} \mathrm{H}$ NMR $\left(\mathrm{CDCl}_{3}\right) \delta($ Major rotamer $): 8.10(\mathrm{~d}$, $1 \mathrm{H}, \mathrm{J}=3.0 \mathrm{~Hz}), 8.02-7.32(\mathrm{~m}, 27 \mathrm{H}), 6.43(\mathrm{~d}, 1 \mathrm{H} \mathrm{J}=3.0 \mathrm{~Hz}), 5.32(\mathrm{~m}, 1 \mathrm{H}), 3.51(\mathrm{~m}, 3 \mathrm{H}), 3.11$ $(\mathrm{m}, 3 \mathrm{H}), 2.32(\mathrm{~m}, 2 \mathrm{H}), 2.08(\mathrm{~m}, 2 \mathrm{H}), 1.96(\mathrm{~m}, 2 \mathrm{H}), 1.70-1.50(\mathrm{~m}, 2 \mathrm{H})$. (Major and minor rotamer together): $8.12(\mathrm{~m}, 0.25 \mathrm{H}), 8.10(\mathrm{~d}, 1 \mathrm{H}, \mathrm{J}=3.0 \mathrm{~Hz}), 8.02-7.32(\mathrm{~m}, 26.75 \mathrm{H}), 6.43(\mathrm{~d}, 1 \mathrm{H}, \mathrm{J}=3.0$ Hz), $5.47(\mathrm{~m}, 0.25 \mathrm{H}), 5.32(\mathrm{~m}) ,.75 \mathrm{H}), 3.63(\mathrm{~m}, 0.25 \mathrm{H}), 3.51(\mathrm{~m}, 2.25 \mathrm{H}), 3.27(\mathrm{~m}, 0.5 \mathrm{H}), 3.16-$ $3.01(\mathrm{~m}, 3 \mathrm{H}), 2.32(\mathrm{~m}, 2 \mathrm{H}), 2.08(\mathrm{~m}, 1.5 \mathrm{H}), 1.96(\mathrm{~m}, 1.5 \mathrm{H}), 1.70-1.50(\mathrm{~m}, 2.5 \mathrm{H}), 1.43-1.30(\mathrm{~m}$,

0.5H). ${ }^{13} \mathrm{C} \mathrm{NMR}\left(\mathrm{CDCl}_{3}\right) \delta 171.8,149.7,148.4,148.3,146.0,143.4,141.5,141.3,141.2,141.0$, $128.7,128.6,127.4,127.3,127.2,127.1,125.8,125.6,125.4,125.3,116.5,108.3,82.1,69.6$, 52.1, 48.9, 31.4, 25.5, 23.7; Rotamer resonances: 172.7, 149.4, 148.1, 147.3, 144.9, 81.5, 69.2, 50.9, 30.4, 23.5. IR (film) 3210, 2972, 1589, 1412, 1144, $696 \mathrm{~cm}^{-1}$. HRMS (ESI, m/z) calcd for $\mathrm{C}_{51} \mathrm{H}_{46} \mathrm{~N}_{3} \mathrm{O}_{2}(\mathrm{M}+\mathrm{H})^{+}$732.3590, found 732.3610. Anal. calcd. for $\mathrm{C}_{51} \mathrm{H}_{45} \mathrm{~N}_{3} \mathrm{O}_{2}$ : C, 83.69; $\mathrm{H}, 6.20$; N, 5.74. Found: C, 83.50; H, 6.23; N, 5.78.

\section{Catalyst 1c}

Prepared as per the synthesis of $\mathbf{1 b}$ using chloropyridine $\mathbf{J}$ (77 $\mathrm{mg}, 0.150 \mathrm{mmol})$, toluene ( $2 \mathrm{~mL}$ ) and pyrrolidine $(125 \mu \mathrm{L}, 1.50 \mathrm{mmol})$. Purification of the resulting product by column chromatography (1:1 Hex- $\mathrm{CH}_{2} \mathrm{Cl}_{2}, \mathrm{R}_{\mathrm{f}}$ 0.1) gave $\mathbf{1 c}(79 \mathrm{mg}, 96 \%)$ as a white solid, m.p. 227-229 ${ }^{\circ} \mathrm{C} .[\alpha]_{\mathrm{D}}{ }^{20}=-78.3\left(\mathrm{c} 0.143, \mathrm{CHCl}_{3}\right) .{ }^{1} \mathrm{H} \mathrm{NMR}\left(\mathrm{CDCl}_{3}\right) \delta$ (Major rotamer): $8.15(\mathrm{~d}, 1 \mathrm{H}, \mathrm{J}=4.0$ Hz), $7.54(\mathrm{~s}, 1 \mathrm{H}), 7.21(\mathrm{~s}, 1 \mathrm{H}), 6.82(\mathrm{~s}, 2 \mathrm{H}), 6.70(\mathrm{~s}, 2 \mathrm{H}), 6.52-6.39(\mathrm{~m}, 3 \mathrm{H}), 5.07(\mathrm{dd}, 1 \mathrm{H}, \mathrm{J}=$ 5.0, $5.5 \mathrm{~Hz}), 3.82(\mathrm{~s}, 6 \mathrm{H}), 3.80(\mathrm{~s}, 6 \mathrm{H}), 3.50(\mathrm{~m}, 3 \mathrm{H}), 3.28-3.09(\mathrm{~m}, 3 \mathrm{H}), 2.28-1.58(\mathrm{~m}, 8 \mathrm{H})$. 
(Major and minor rotamer together): $8.15(\mathrm{~d}, 1 \mathrm{H}, \mathrm{J}=4.0 \mathrm{~Hz}), 7.58-7.54(\mathrm{~m}, 1 \mathrm{H}), 7.21(\mathrm{~s}, 1 \mathrm{H})$, $6.82(\mathrm{~s}, 2 \mathrm{H}), 6.70(\mathrm{~s}, 2 \mathrm{H}), 6.52-6.39(\mathrm{~m}, 3 \mathrm{H}), 5.07(\mathrm{dd}, 1 \mathrm{H}, \mathrm{J}=5.0,5.5 \mathrm{~Hz}), 3.82(\mathrm{~s}, 6 \mathrm{H}), 3.80(\mathrm{~s}$, $6 \mathrm{H}), 3.65(\mathrm{~m}, 0.15 \mathrm{H}), 3.50(\mathrm{~m}, 2.55 \mathrm{H}), 3.28-3.09(\mathrm{~m}, 3 \mathrm{H}), 2.91(\mathrm{~m}, 0.3 \mathrm{H}), 2.28-1.58(\mathrm{~m}, 8 \mathrm{H})$. ${ }^{13} \mathrm{C} \mathrm{NMR}\left(\mathrm{CDCl}_{3}\right) \delta 171.7,160.3,160.1,149.6,148.5,148.4,147.3,144.8,116.7,108.3,106.2$, 106.1, 99.1, 99.0, 81.7, 68.9, 55.3, 55.2, 52.1, 48.8, 30.7, 25.5, 23.6; Rotamer resonances: 172.9, 149.2, 146.9, 145.1, 105.8, 98.8, 80.9, 70.4, 51.4, 31.4, 22.5. IR (film) 3217, 2955, 2332, 1592, 1424, $1153 \mathrm{~cm}^{-1}$. HRMS (ESI, m/z) calcd for $\mathrm{C}_{31} \mathrm{H}_{38} \mathrm{~N}_{3} \mathrm{O}_{6}(\mathrm{M}+\mathrm{H})^{+}$548.2761, found 548.2775. Anal. calcd. for $\mathrm{C}_{31} \mathrm{H}_{37} \mathrm{~N}_{3} \mathrm{O}_{6}$ : C, 67.99; H, 6.81; N, 7.67. Found: C, 67.78; H, 6.78; N, 7.48.

\section{Catalyst 1d}

Prepared as per the synthesis of $\mathbf{1 b}$ using chloropyridine K (80 $\mathrm{mg}, 0.179 \mathrm{mmol})$, toluene (2 $\mathrm{mL})$ and pyrrolidine $(149 \mu \mathrm{L}, 1.79 \mathrm{mmol})$. Purification of the resulting product by column chromatography (1:1 $\mathrm{CH}_{2} \mathrm{Cl}_{2}$-EtOAc, $\left.\mathrm{R}_{\mathrm{f}} 0.2\right)$ gave $\mathbf{1 d}(83 \mathrm{mg}, 96 \%)$ as a white solid, m.p. 179$180{ }^{\circ} \mathrm{C} .[\alpha]_{\mathrm{D}}{ }^{20}=-50.3\left(\mathrm{c} 0.171, \mathrm{CHCl}_{3}\right) .{ }^{1} \mathrm{H} \mathrm{NMR}\left(\mathrm{CDCl}_{3}\right) \delta($ Major rotamer $): 8.14(\mathrm{~d}, 1 \mathrm{H}, \mathrm{J}=4.0$ Hz), $7.45(\mathrm{~s}, 1 \mathrm{H}), 7.25(\mathrm{~s}, 2 \mathrm{H}), 7.11(\mathrm{~s}, 2 \mathrm{H}), 7.06(\mathrm{~s}, 1 \mathrm{H}), 6.94(\mathrm{~s}, 1 \mathrm{H}), 6.86(\mathrm{~s}, 1 \mathrm{H}), 6.45(\mathrm{~d}, 1 \mathrm{H}$, $\mathrm{J}=4.0 \mathrm{~Hz}), 5.20(\mathrm{dd}, 1 \mathrm{H}, \mathrm{J}=5.3 \mathrm{~Hz}), 3.50(\mathrm{~m}, 3 \mathrm{H}), 3.11(\mathrm{~m}, 2 \mathrm{H}), 2.94(\mathrm{~m}, 1 \mathrm{H}), 2.39(\mathrm{~s}, 6 \mathrm{H})$, $2.32(\mathrm{~s}, 6 \mathrm{H}), 2.26-1.52(\mathrm{~m}, 8 \mathrm{H})$. (Major and minor rotamer together): $8.14(\mathrm{~d}, 1 \mathrm{H}, \mathrm{J}=3.5 \mathrm{~Hz})$, $7.45(\mathrm{~s}, 1 \mathrm{H}), 7.25(\mathrm{~s}, 2 \mathrm{H}), 7.16-7.06(\mathrm{~m}, 3 \mathrm{H}), 6.96-6.85(\mathrm{~m}, 2 \mathrm{H}), 6.45(\mathrm{~d}, 1 \mathrm{H}, \mathrm{J}=4.0 \mathrm{~Hz}), 5.26-$ $5.20(\mathrm{dd}, 1 \mathrm{H}, \mathrm{J}=5.3,5.3 \mathrm{~Hz}), 3.50(\mathrm{~m}, 2.8 \mathrm{H}), 3.33(\mathrm{~m}, 0.2 \mathrm{H}), 3.11(\mathrm{~m}, 2 \mathrm{H}), 2.94(\mathrm{~m}, 1 \mathrm{H}), 2.39$ (s, 6H), $2.32(\mathrm{~s}, 6 \mathrm{H}), 2.26-1.52(\mathrm{~m}, 8 \mathrm{H}) .{ }^{13} \mathrm{C} \mathrm{NMR}\left(\mathrm{CDCl}_{3}\right) \delta 171.2,149.4,148.4,148.3,145.0$, $142.5,137.2,136.9,128.9,128.8,125.5,125.4,116.7,108.3,82.1,68.2,51.8,48.8,30.4,25.5$, 23.6, 21.5, 21.4; Rotamer resonances: 171.5, 125.7, 82.1, 68.8, 49.0, 29.9, 25.6, 22.5. IR (film) 3244, 2970, 1587, 1415, $749 \mathrm{~cm}^{-1}$. HRMS (ESI, m/z) calcd for $\mathrm{C}_{31} \mathrm{H}_{38} \mathrm{~N}_{3} \mathrm{O}_{2}(\mathrm{M}+\mathrm{H})^{+} 484.2964$, 
found 484.2964. Anal. calcd. for $\mathrm{C}_{31} \mathrm{H}_{37} \mathrm{~N}_{3} \mathrm{O}_{2}$ : C, 76.98; H, 7.71; N, 8.69. Found: C, 76.84; H, $7.73 ; \mathrm{N}, 8.53$.

\section{Catalyst 1e}

Prepared as per the synthesis of $\mathbf{1 b}$ using chloropyridine $\mathbf{L}$ (55 $\mathrm{mg}, 0.083 \mathrm{mmol})$, toluene (2 $\mathrm{mL})$ and pyrrolidine $(70 \mu \mathrm{L}, 0.828 \mathrm{mmol})$. Purification of the resulting product by column chromatography $\left(1: 1 \mathrm{CH}_{2} \mathrm{Cl}_{2}, \mathrm{R}_{\mathrm{f}} 0.2\right)$ gave $1 \mathrm{e}(55 \mathrm{mg}, 95 \%)$ as a white solid, m.p. $120-122{ }^{\circ} \mathrm{C}$. $[\alpha]_{\mathrm{D}}{ }^{20}=-54\left(\mathrm{c} 0.1, \mathrm{CHCl}_{3}\right) .{ }^{1} \mathrm{H} \mathrm{NMR}\left(\mathrm{CDCl}_{3}\right) \delta$ (Major and minor rotamers): 8.22-7.83 (m, 7H), $7.44(\mathrm{~s}, 1 \mathrm{H}), 6.49(\mathrm{~d}, 1 \mathrm{H}, \mathrm{J}=5.5 \mathrm{~Hz}), 5.24-5.05(\mathrm{~m}, 1 \mathrm{H}), 3.68(\mathrm{~m}, 1 \mathrm{H}), 3.49(\mathrm{~m}, 2 \mathrm{H}), 3.28-2.91$ (m, 3H), 2.28-1.65 (m, 8H). ${ }^{13} \mathrm{C} \mathrm{NMR}\left(\mathrm{CDCl}_{3}\right) \delta 172.4,149.9,148.6,147.9,146.3,144.5,131.7$, $131.6,127.5,127.4,123.1,123.0,122.1,122.0,115.6,108.6,80.4,68.9,52.2,49.1,31.2,25.4$, 23.7. Rotamer resonances: $173.1,149.6,147.3,80.2,68.7,50.9,30.4,29.5,22.5$. IR (film) 3240, 2928, 2298, 1642, 1371, 1132, $682 \mathrm{~cm}^{-1}$. HRMS (ESI, m/z) calcd for $\mathrm{C}_{31} \mathrm{H}_{26} \mathrm{~F}_{12} \mathrm{~N}_{3} \mathrm{O}_{2}(\mathrm{M}+\mathrm{H})^{+}$ 700.1833, found 700.1835. Anal. calcd. for $\mathrm{C}_{31} \mathrm{H}_{25} \mathrm{~F}_{12} \mathrm{~N}_{3} \mathrm{O}_{2}$ : C, 53.23; H, 3.60; F, 32.59, N, 6.01. Found: C, 53.07; H, 3.71; F, 32.29, N, 5.72.

One pot synthesis and resolution of 9

A $1 \mathrm{~mL}$ reaction vessel charged with $1 \mathrm{e}(12.7 \mathrm{mg}, 18.2 \mu \mathrm{mol})$ and a small magnetic stirring bar was placed under an atmosphere of Ar. To this was added benzaldehyde (370 $\mu \mathrm{L}, 0.364 \mathrm{mmol})$, DBU $(380 \mu \mathrm{L}, 0.255 \mathrm{mmol})$ and methyl acrylate $(985 \mu \mathrm{L}, 1.09 \mathrm{mmol})$ via syringe and the resulting homogeneous solution stirred at room temperature for $24 \mathrm{~h} . \mathrm{CH}_{2} \mathrm{Cl}_{2}(500 \mu \mathrm{L})$ was then added via syringe and the solution was cooled to $-78{ }^{\circ} \mathrm{C}$ and left to stir for 30 minutes. Isobutyric anhydride $(91 \mu \mathrm{L}, 0.546 \mathrm{mmol})$ was subsequently added via syringe. After $24 \mathrm{~h}$ at $-78^{\circ} \mathrm{C}$ 
the reaction was quenched by the addition of $\mathrm{MeOH}(200 \mu \mathrm{L})$ and allowed to warm to ambient temperature. Solvents were removed in vacuo. The alcohol and its ester were separated from the catalyst by passing a concentrated solution of the crude $\left(\mathrm{CH}_{2} \mathrm{Cl}_{2}\right)$ through a pad of silica gel. The selectivity of the kinetic resolution $(s=4.3)$ was then established by CSP-HPLC on a Chiralcel AS-H column (4.6 x $250 \mathrm{~mm})$, hexanes/i-PrOH, 90/10, $1 \mathrm{~mL} \mathrm{~min}^{-1}$, RT, UV detection at $220 \mathrm{~nm}$ for 9a and a Chiralcel OD-H column (4.6 x $250 \mathrm{~mm})$, hexanes/i-PrOH, 90/10, $1 \mathrm{~mL} \mathrm{~min}^{-1}$, RT, UV detection at $220 \mathrm{~nm}$ for 9b. Retention times: 9a $(75 \%$ ee): $7.8 \mathrm{~min},(R)$-isomer (minor) and $14.9 \mathrm{~min},(S)$-isomer (major), ${ }^{6} \mathbf{9 b}(35 \%$ ee $)$ : $6.0 \mathrm{~min},(S)$-isomer (minor) and $6.9 \mathrm{~min}(R)$-isomer (major). The alcohol was then isolated by column chromatography $\left(\mathrm{CH}_{2} \mathrm{Cl}_{2}\right)$ to give $(\boldsymbol{S})-9 \mathbf{a}(17.6$ $\mathrm{mg}, 25 \%, 75 \%$ ee $)$ as a colorless oil. $[\alpha]_{\mathrm{D}}{ }^{20}=+47.3\left(\mathrm{c} 0.1, \mathrm{CHCl}_{3}\right) .{ }^{1} \mathrm{H}_{\mathrm{NMR}}\left(\mathrm{CDCl}_{3}\right) \delta 7.41-7.31$ $(\mathrm{m}, 5 \mathrm{H}), 6.37(\mathrm{~s}, 1 \mathrm{H}), 5.85(\mathrm{~s}, 1 \mathrm{H}), 5.59(\mathrm{~s}, 1 \mathrm{H}), 3.76(\mathrm{~s}, 3 \mathrm{H}), 3.02(\mathrm{bs}, 1 \mathrm{H})$. Absolute configuration of the major isomer determined by comparision of optical rotation ${ }^{7}$ and CSPHPLC data ${ }^{6}$ with that in the literature.

One pot synthesis and resolution of $\mathbf{1 0}$

A $1 \mathrm{~mL}$ reaction vessel charged with $1 \mathrm{e}(4.3 \mathrm{mg}, 6.14 \mu \mathrm{mol})$ and a small magnetic stirring bar was placed under an atmosphere of Ar. To this was added $o$-anisaldehyde (50 $\mathrm{mg}, 0.368 \mathrm{mmol})$, DBU $(18 \mu \mathrm{L}, 0.123 \mathrm{mmol})$ and methyl acrylate $(11 \mu \mathrm{L}, 0.123 \mathrm{mmol})$ via syringe and the resulting homogeneous solution stirred at $\mathrm{rt}$ for $96 \mathrm{~h} . \mathrm{CH}_{2} \mathrm{Cl}_{2}(500 \mu \mathrm{L})$ was then added via syringe and the solution was cooled to $-78^{\circ} \mathrm{C}$ and left to stir for $30 \mathrm{~min}$. Isobutyric anhydride (16 $\mu \mathrm{L}, 0.98 \mathrm{mmol}$ ) was subsequently added via syringe. After $24 \mathrm{~h}$ at $-78{ }^{\circ} \mathrm{C}$ the reaction was quenched by the addition of $\mathrm{MeOH}(200 \mu \mathrm{L})$ and allowed to warm to ambient temperature. Solvents were removed in vacuo. The alcohol and its ester were separated from the catalyst by 
passing a concentrated solution of the crude $\left(\mathrm{CH}_{2} \mathrm{Cl}_{2}\right)$ through a pad of silica gel. The selectivity of the kinetic resolution $(s=7.6)$ was then established by CSP-HPLC on a Chiralcel OD-H column (4.6 x $250 \mathrm{~mm}$ ), hexanes/i-PrOH, 98/2, $1 \mathrm{~mL} \mathrm{~min}^{-1}$, RT, UV detection at $220 \mathrm{~nm}$. Retention times: 10a (89\% ee): $34.8 \mathrm{~min},(S)$-isomer (major) and $45.0 \mathrm{~min},(R)$-isomer (minor), 10b $(47 \%$ ee): $8.5 \mathrm{~min},(S)$-isomer (minor) and $22.3 \mathrm{~min}(R)$-isomer (major). The alcohol was then isolated by column chromatography $\left(\mathrm{CH}_{2} \mathrm{Cl}_{2}\right)$ to give $(\mathbf{S})-\mathbf{1 0 a}(6.9 \mathrm{mg}, 25 \%, 89 \%$ ee $)$ as a colorless oil $[\alpha]_{\mathrm{D}}^{20}=+83\left(\mathrm{c} 0.1, \mathrm{CHCl}_{3}\right) .{ }^{1} \mathrm{H} \mathrm{NMR}\left(\mathrm{CDCl}_{3}\right) \delta 7.36(\mathrm{~d}, 1 \mathrm{H}, \mathrm{J}=7.5 \mathrm{~Hz}), 7.28(\mathrm{t}$, 1H, J $8.0 \mathrm{~Hz}$ ), 6.97 (app. t, 1H, J = 7.5 Hz), 6.89 (d, 1H, J = $8.0 \mathrm{~Hz}), 6.31(\mathrm{~s}, 1 \mathrm{H}), 5.88(\mathrm{~s}, 1 \mathrm{H})$, $5.73(\mathrm{~s}, 1 \mathrm{H}), 3.84(\mathrm{~s}, 3 \mathrm{H}), 3.75(\mathrm{~s}, 3 \mathrm{H})$. Absolute configuration is tentatively assigned based on a comparison of CSP-HPLC retention times and optical rotation data with that of $(\boldsymbol{S})-\mathbf{9 a}$.

\subsection{Spectral data for previously uncharacterized esters}

Isobutyric ester 9b: Isolated $(47.3 \mathrm{mg}, 49.5 \%, 35 \% e e)$ as a colorless oil. $[\alpha]_{\mathrm{D}}{ }^{20}=-33$ (c 0.16 , $\left.\mathrm{CHCl}_{3}\right) .{ }^{1} \mathrm{H} \mathrm{NMR}\left(\mathrm{CDCl}_{3}\right) \delta$ 7.42-7.30 (m, 5H), $6.69(\mathrm{~s}, 1 \mathrm{H}), 6.41(\mathrm{~s}, 1 \mathrm{H}), 5.86(\mathrm{~s}, 1 \mathrm{H}), 3.74(\mathrm{~s}$, 3H), 2.63 (app. hept, $1 \mathrm{H}, \mathrm{J}=7.0 \mathrm{~Hz}), 1.22-1.17(\mathrm{~m}, 6 \mathrm{H}) .{ }^{13} \mathrm{C}$ NMR $\left(\mathrm{CDCl}_{3}\right) \delta 175.2,165.4$, 139.9, 137.9, 128.3, 128.1, 127.4, 125.6, 72.6, 51.8, 33.9, 18.8, 18.6. IR (film) 2975, 1729, 1495, $1249,1140 \mathrm{~cm}^{-1}$. HRMS (ESI, m/z) calcd for $\mathrm{C}_{15} \mathrm{H}_{18} \mathrm{O}_{4} \mathrm{Na}(\mathrm{M}+\mathrm{Na})^{+} 285.1103$, found 285.1115 .

Isobutyric ester 10b: Isolated $(16.2 \mathrm{mg}, 45.1 \%, 47 \% e e)$ as a colorless oil. $[\alpha]_{\mathrm{D}}{ }^{20}=-62(\mathrm{c} 0.1$, $\left.\mathrm{CHCl}_{3}\right) .{ }^{1} \mathrm{H} \mathrm{NMR}\left(\mathrm{CDCl}_{3}\right) \delta$ 7.34-7.27 (m, 2H), 7.05 (s, 1H), 6.96(app.dd, 1H, J = 7.5 Hz), 6.90 $(\mathrm{d}, 1 \mathrm{H}, \mathrm{J}=8.0 \mathrm{~Hz}), 6.41(\mathrm{~s}, 1 \mathrm{H}), 5.61(\mathrm{~s}, 1 \mathrm{H}), 3.84(\mathrm{~s}, 3 \mathrm{H}), 3.76$ (s, 3H), 2.63 (app. hept, 1H, J = 7.0 Hz), 1.22-1.18 (m, 6H). ${ }^{13} \mathrm{C} \mathrm{NMR}\left(\mathrm{CDCl}_{3}\right)$ 8: 175.0, 165.4, 156.5, 138.8, 129.0, 127.1, 126.8, 
125.7, 119.9, 110.3, 67.2, 56.0, 51.5, 33.6, 18.5, 18.4. IR (film) 2973, 2840, 1725, 1492, 1246 $\mathrm{cm}^{-1}$. HRMS (ESI, m/z) calcd for $\mathrm{C}_{16} \mathrm{H}_{20} \mathrm{O}_{5} \mathrm{Na}(\mathrm{M}+\mathrm{Na})^{+} 315.1208$, found 315.1216.

Isobutyric ester 11b: Isolated $(40.1 \mathrm{mg}, 75 \%)$ as a colorless oil. $[\alpha]_{\mathrm{D}}{ }^{20}=-14.2\left(\mathrm{c} 0.29, \mathrm{CHCl}_{3}\right)$. ${ }^{1} \mathrm{H}$ NMR $\left(\mathrm{CDCl}_{3}\right) \delta$ 7.42-7.30 (m, 5H), 6.35 (s, 1H), 6.10 (s, 1H), 6.01 (s, 1H), 2.71 (app. hept, $1 \mathrm{H}, \mathrm{J}=7.0 \mathrm{~Hz}), 1.28-1.22(\mathrm{~m}, 6 \mathrm{H}) .{ }^{13} \mathrm{C} \mathrm{NMR}\left(\mathrm{CDCl}_{3}\right) \delta: 174.9,135.3,131.5,128.7,128.5$, 126.4, 123.0, 115.7, 73.6, 33.5, 18.4, 18.3. IR (film) 2976, 2228, 1739, 1496, $1243 \mathrm{~cm}^{-1}$. HRMS (ESI, m/z) calcd for $\mathrm{C}_{14} \mathrm{H}_{15} \mathrm{NO}_{2} \mathrm{Na}(\mathrm{M}+\mathrm{Na})^{+} 252.1000$, found 252.1009 .

Isobutyric ester 12b: Isolated (36 mg, $59.3 \%)$ as a colorless oil. $[\alpha]_{D}{ }^{20}=-11.9\left(\mathrm{c} 0.26, \mathrm{CHCl}_{3}\right)$. ${ }^{1} \mathrm{H}$ NMR $\left(\mathrm{CDCl}_{3}\right) \delta 7.48$ (d, 1H, J =7.5 Hz), 7.35 (app.dd, 1H, J = $8.0 \mathrm{~Hz}$ ), 7.04 (app.dd, 1H, J = $7.5 \mathrm{~Hz}), 6.92(\mathrm{~d}, 1 \mathrm{H}, \mathrm{J}=8.0 \mathrm{~Hz}), 6.73(\mathrm{~s}, 1 \mathrm{H}), 6.03(\mathrm{~s}, 1 \mathrm{H}), 6.00$ (s, 1H), 3.87 (s, 3H), 2.71 (app. hept, $1 \mathrm{H}, \mathrm{J}=7.0 \mathrm{~Hz}), 1.28-1.23(\mathrm{~m}, 6 \mathrm{H}) .{ }^{13} \mathrm{C} \mathrm{NMR}\left(\mathrm{CDCl}_{3}\right) \delta: 174.9,155.8,131.5,129.7,126.4$, 123.9, 122.2, 120.5, 115.9, 110.2, 68.2, 55.0, 33.6, 18.5, 18.4. IR (film) 2975, 2841, 2228, 1740, 1492, 1247, $1142 \mathrm{~cm}^{-1}$. HRMS (ESI, m/z) calcd for $\mathrm{C}_{15} \mathrm{H}_{17} \mathrm{NO}_{3} \mathrm{Na}(\mathrm{M}+\mathrm{Na})^{+} 282.1106$, found 282.1118 . 


\subsection{HPLC conditions/retention times and optical rotation data}

(cis)-4-Dimethylamino-benzoic acid 2-hydroxy-cyclohexyl ester (2)



Chiralcel OD-H (4.6 mm x $25 \mathrm{~cm}$ ), hexanes/i-PrOH, 90/10, $1 \mathrm{~mL} \mathrm{~min}^{-1}$, RT, UV detection at 220 nm. Retention times: $17.3 \min (1 R, 2 S)$ minor and $34.4 \min (1 S, 2 R)$ major. $^{8}$

(cis)-4-Dimethylamino-benzoic acid 2-isobutyryloxy-cyclohexyl ester<smiles>CC(C)C(=O)OC1CCCCC1OC(=O)c1ccc(N(C)C)cc1</smiles>

Chiralcel OD-H column (4.6 x $250 \mathrm{~mm}$ ), hexanes/i-PrOH, 90/10, $1 \mathrm{~mL} \mathrm{m^{-1 }}$, RT, UV detection at $220 \mathrm{~nm}$. Retention times: $8.4 \min (1 S, 2 R)$ minor and $9.9 \min (1 R, 2 S)$ major. $^{8}$

\section{2-Methyl-1-phenyl-propan-1-ol}



Chiralcel OD-H (4.6 mm x $25 \mathrm{~cm}$ ), hexanes $/ i-\mathrm{PrOH}, 99 / 1,1 \mathrm{~mL} \mathrm{~min}^{-1}$, RT, UV detection at 220 $\mathrm{nm}$. Retention times: $21.2 \mathrm{~min}(R)$ major and $22.8 \min (S)$ minor. $^{9}$ 


\section{Isobutyric acid 2-methyl-1-phenyl-propyl ester}

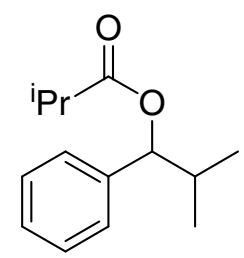

Chiralcel OD-H (4.6 mm x $25 \mathrm{~cm}$ ), hexanes/i-PrOH, 99.6/0.4, $1 \mathrm{~mL} \mathrm{~min}^{-1}$, RT, UV detection at $220 \mathrm{~nm}$. Retention times: $5.4 \mathrm{~min}(R)$ minor and $5.9 \min (S)$ major. ${ }^{9}$

(trans)-4-Dimethylamino-benzoic acid 2-hydroxy-cyclohexyl ester (8)<smiles>CN(C)c1ccc(C(=O)OC2CCCCC2O)cc1</smiles>

Chiralcel AS-H (4.6 mm x $25 \mathrm{~cm}$ ), hexanes/i-PrOH, 90/10, $1 \mathrm{~mL} \mathrm{~min}^{-1}$, RT, UV detection at 220 $\mathrm{nm}$. Retention times: $12.2 \mathrm{~min}$ (minor) and $25.8 \mathrm{~min}$ (major).

(trans)-4-Dimethylamino-benzoic acid 2-isobutyryloxy-cyclohexyl ester<smiles>CC(C)C(=O)OC1CCCCC1OC(=O)c1ccc(N(C)C)cc1</smiles>

Chiralcel OD-H (4.6 mm x $25 \mathrm{~cm}$ ), hexanes/i-PrOH, 90/10, $1 \mathrm{~mL} \mathrm{~min}^{-1}$, RT, UV detection at 220 $\mathrm{nm}$. Retention times: $11.9 \mathrm{~min}$ (major) and $14.7 \mathrm{~min}$ (minor). 
<smiles>C=C(C(=O)OC)C(O)c1ccccc1</smiles>

Chiralcel AS-H column (4.6 x $250 \mathrm{~mm}$ ), hexanes/i-PrOH, 90/10, $1 \mathrm{~mL} \mathrm{~min}^{-1}$, RT, UV detection at $220 \mathrm{~nm}$. Retention times: $7.8 \mathrm{~min},(R)$-isomer (minor) and $14.9 \mathrm{~min},(S)$-isomer (major). ${ }^{6}$

\section{2-(Isobutyryloxy-phenyl-methyl)-acrylic acid methyl ester (9b)}<smiles>C=C(C(=O)OC)C(OC(=O)CC)c1ccccc1</smiles>

Chiralcel OD-H column (4.6 x $250 \mathrm{~mm}$ ), hexanes/i-PrOH, 90/10, $1 \mathrm{~mL} \mathrm{~min}^{-1}$, RT, UV detection at $220 \mathrm{~nm}$. Retention times: $6.0 \mathrm{~min},(S)$-isomer (minor) and $6.7 \mathrm{~min},(R)$-isomer (major). $[\alpha]_{\mathrm{D}}{ }^{20}$ $=-33\left(\mathrm{c} 0.16, \mathrm{CHCl}_{3}\right)$.

\section{2-[Hydroxy-(2-methoxy-phenyl)-methyl]-acrylic acid methyl ester (10a)}<smiles>C=C(C(=O)OC)C(O)c1ccccc1OC</smiles>

Chiralcel OD-H column (4.6 x $250 \mathrm{~mm}$ ), hexanes/i-PrOH, 98/2, $1 \mathrm{~mL} \mathrm{~min}^{-1}$, RT, UV detection at $220 \mathrm{~nm}$. Retention times: $34.8 \mathrm{~min},(S)$-isomer (major) and $45.0 \mathrm{~min},(R)$-isomer (minor). 
<smiles>COC(=O)C(=O)C(OC(=O)C(C)C)c1ccccc1OC</smiles>

Chiralcel OD-H column (4.6 x $250 \mathrm{~mm}$ ), hexanes/i-PrOH, 98/2, $1 \mathrm{~mL} \mathrm{~min}^{-1}$, RT, UV detection at $220 \mathrm{~nm}$. Retention times: $8.5 \mathrm{~min},(S)$-isomer (minor) and $22.3 \mathrm{~min},(R)$-isomer (major). $[\alpha]_{\mathrm{D}}{ }^{20}=$ $-62\left(\mathrm{c} 0.1, \mathrm{CHCl}_{3}\right)$.

2-(Hydroxy-phenyl-methyl)-acrylonitrile (11a)<smiles>C=C(C#N)C(O)c1ccccc1</smiles>

Chiralcel OD-H column (4.6 x $250 \mathrm{~mm}$ ), hexanes/i-PrOH, 90/10, $1 \mathrm{~mL} \mathrm{~min}^{-1}$, RT, UV detection at $220 \mathrm{~nm}$. Retention times: $13.2 \mathrm{~min}$ (minor) and $14.0 \mathrm{~min}$ (major). $[\alpha]_{\mathrm{D}}{ }^{20}=+77$ (c .04, $\mathrm{CHCl}_{3}$ ).

\section{2-(Isobutyryloxy-phenyl-methyl)-acrylonitrile (11b)}<smiles>C=C(C#N)C(OC(=O)CC)c1ccccc1</smiles>

Could not be resolved by CSP-HPLC using Chiralcel OD-H, Chiralcel AS-H or Chiralpak AD-H columns.

$[\alpha]_{\mathrm{D}}^{20}=-14.2\left(\mathrm{c} 0.29, \mathrm{CHCl}_{3}\right)$. 


\section{2-[Hydroxy-(2-methoxy-phenyl)-methyl]-acrylonitrile (12a)}

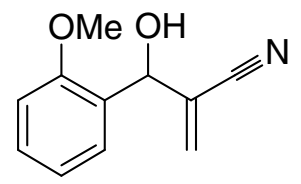

Chiralcel OD-H column (4.6 x $250 \mathrm{~mm}$ ), hexanes/i-PrOH, 90/10, $1 \mathrm{~mL} \mathrm{~min}^{-1}$, RT, UV detection at $220 \mathrm{~nm}$. Retention times: $11.3 \mathrm{~min},(R)$-isomer (major) and $12.6 \mathrm{~min},(S)$-isomer (minor). ${ }^{10}$ Note that due to a priority change, the label of the stereogenic centre changes in (+)-12a from $(S)$ to $(R)$. $[\alpha]_{\mathrm{D}}{ }^{20}=+31.8\left(\mathrm{c} 0.08, \mathrm{CHCl}_{3}\right)$. Absolute configuration of the major isomer determined by comparision of optical rotation data with that in the literature. ${ }^{10}$

\section{2-[Isobutyryloxy-(2-methoxy-phenyl)-methyl]-acrylonitrile (12b)}

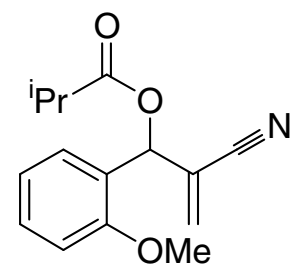

Could not be resolved by CSP-HPLC using Chiralcel OD-H, Chiralcel AS-H or Chiralpak AD-H columns.

$[\alpha]_{\mathrm{D}}^{20}=-11.9\left(\mathrm{c} 0.26, \mathrm{CHCl}_{3}\right)$. 


\subsection{NMR spectra}

${ }^{1} \mathrm{H}$ NMR spectrum $\left(400 \mathrm{MHz}, \mathrm{CDCl}_{3}\right)$ of catalyst $\mathbf{1 b}$

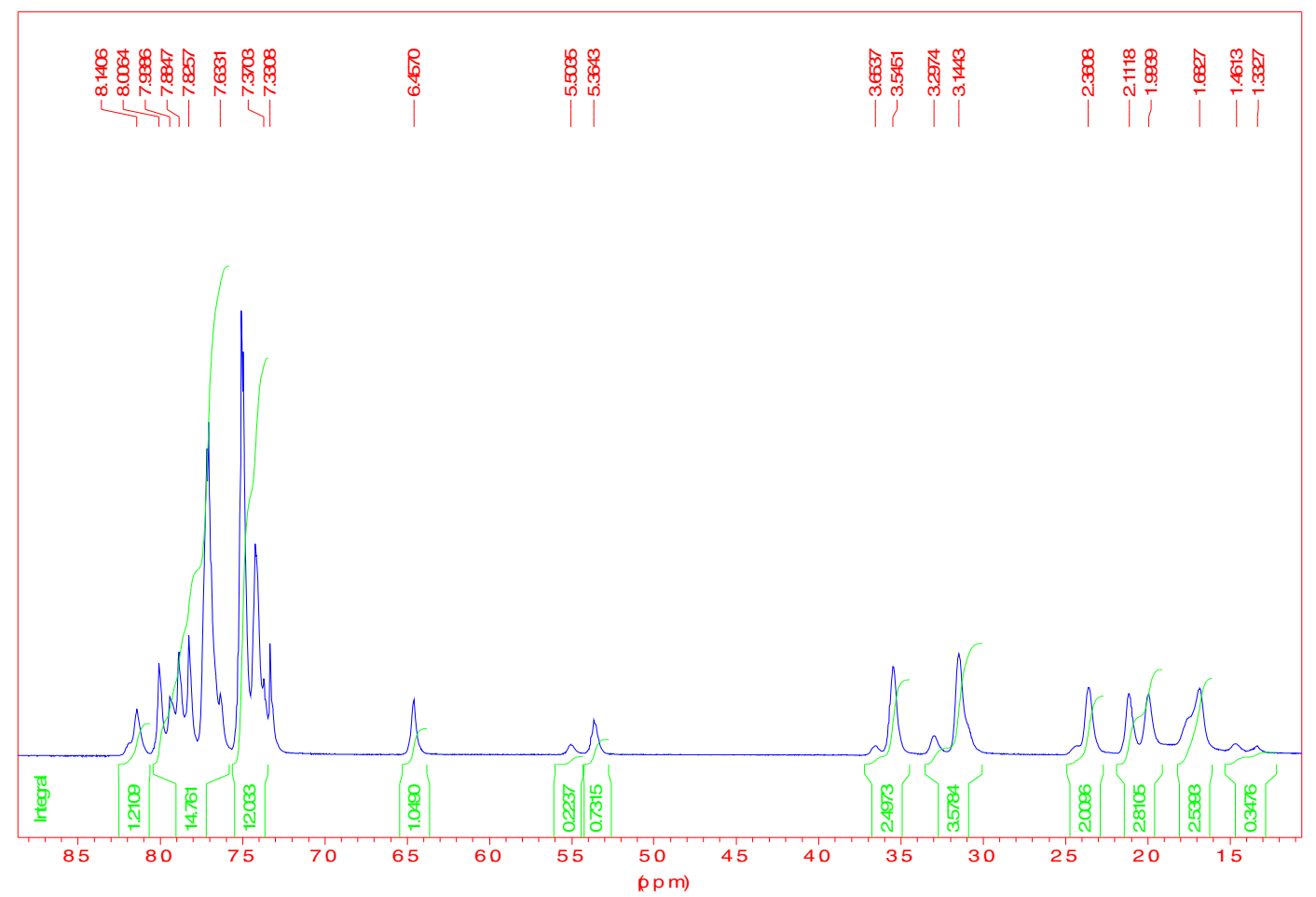

${ }^{13} \mathrm{C}$ NMR spectrum $\left(100 \mathrm{MHz}, \mathrm{CDCl}_{3}\right)$ of catalyst $\mathbf{1 b}$

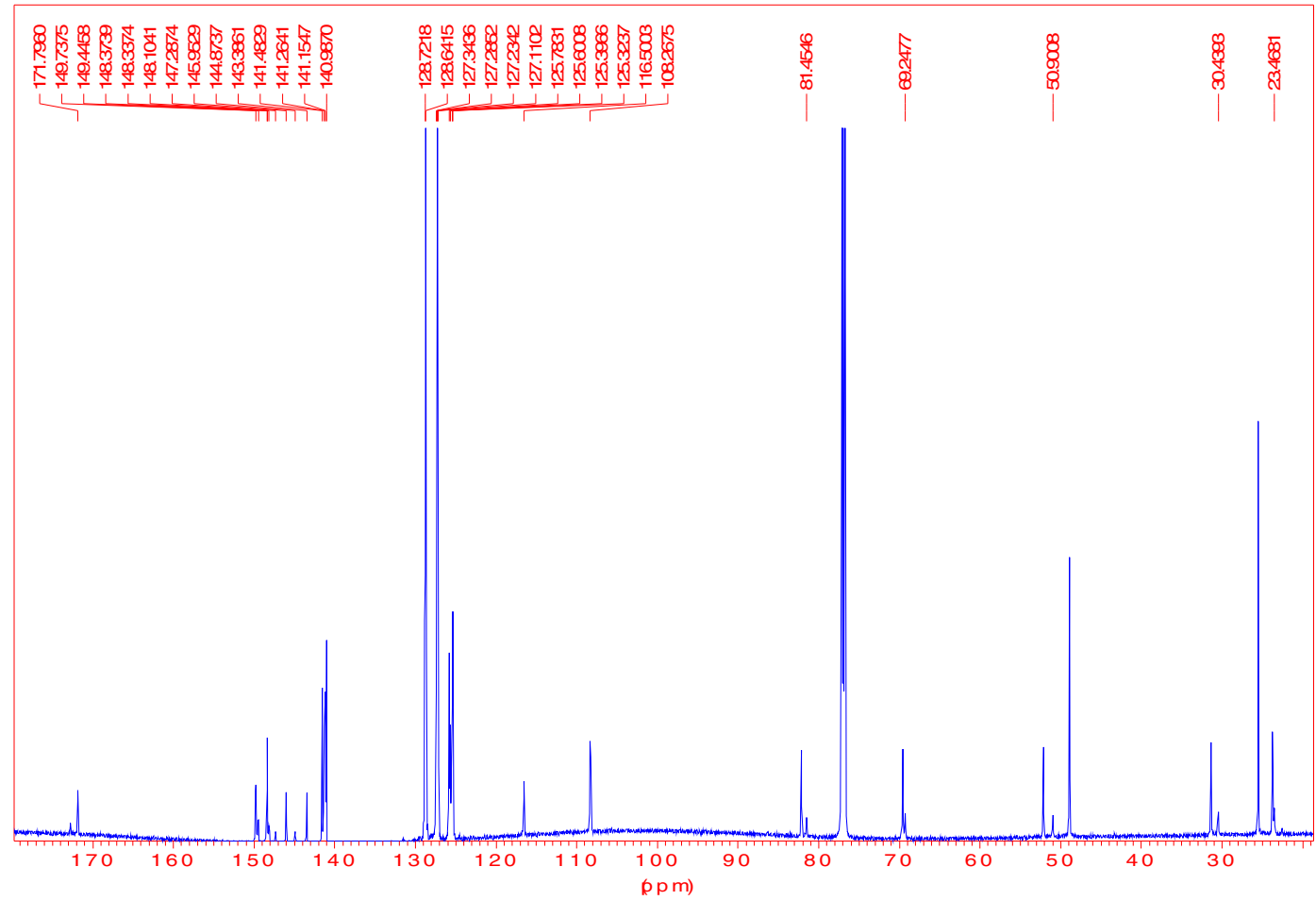


${ }^{1} \mathrm{H}$ NMR spectrum $\left(400 \mathrm{MHz}, \mathrm{CDCl}_{3}\right)$ of catalyst $\mathbf{1 c}$



${ }^{13} \mathrm{C}$ NMR spectrum $\left(100 \mathrm{MHz}, \mathrm{CDCl}_{3}\right)$ of catalyst $\mathbf{1 c}$

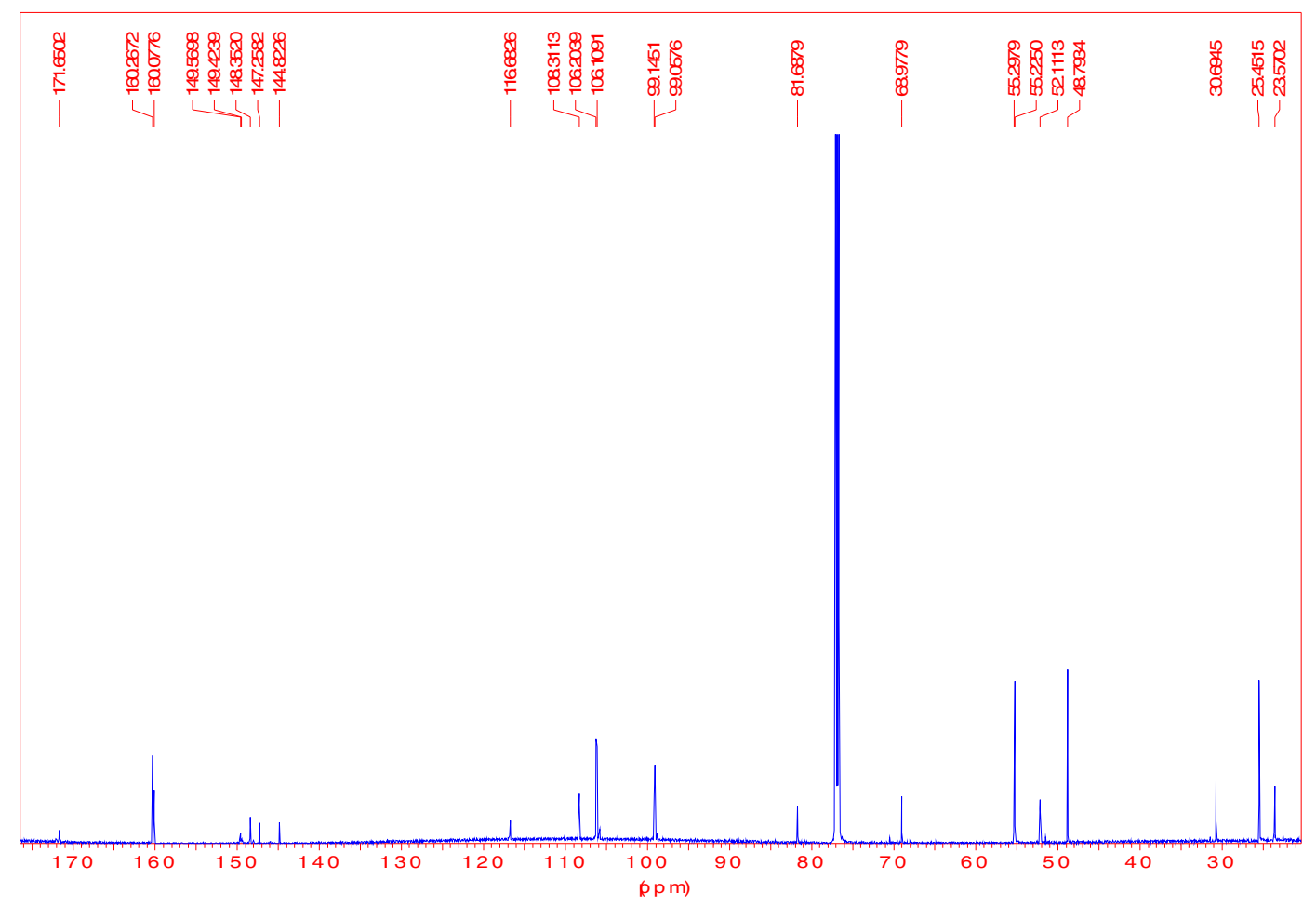


${ }^{1} \mathrm{H}$ NMR spectrum $\left(400 \mathrm{MHz}, \mathrm{CDCl}_{3}\right)$ of catalyst $\mathbf{1 d}$

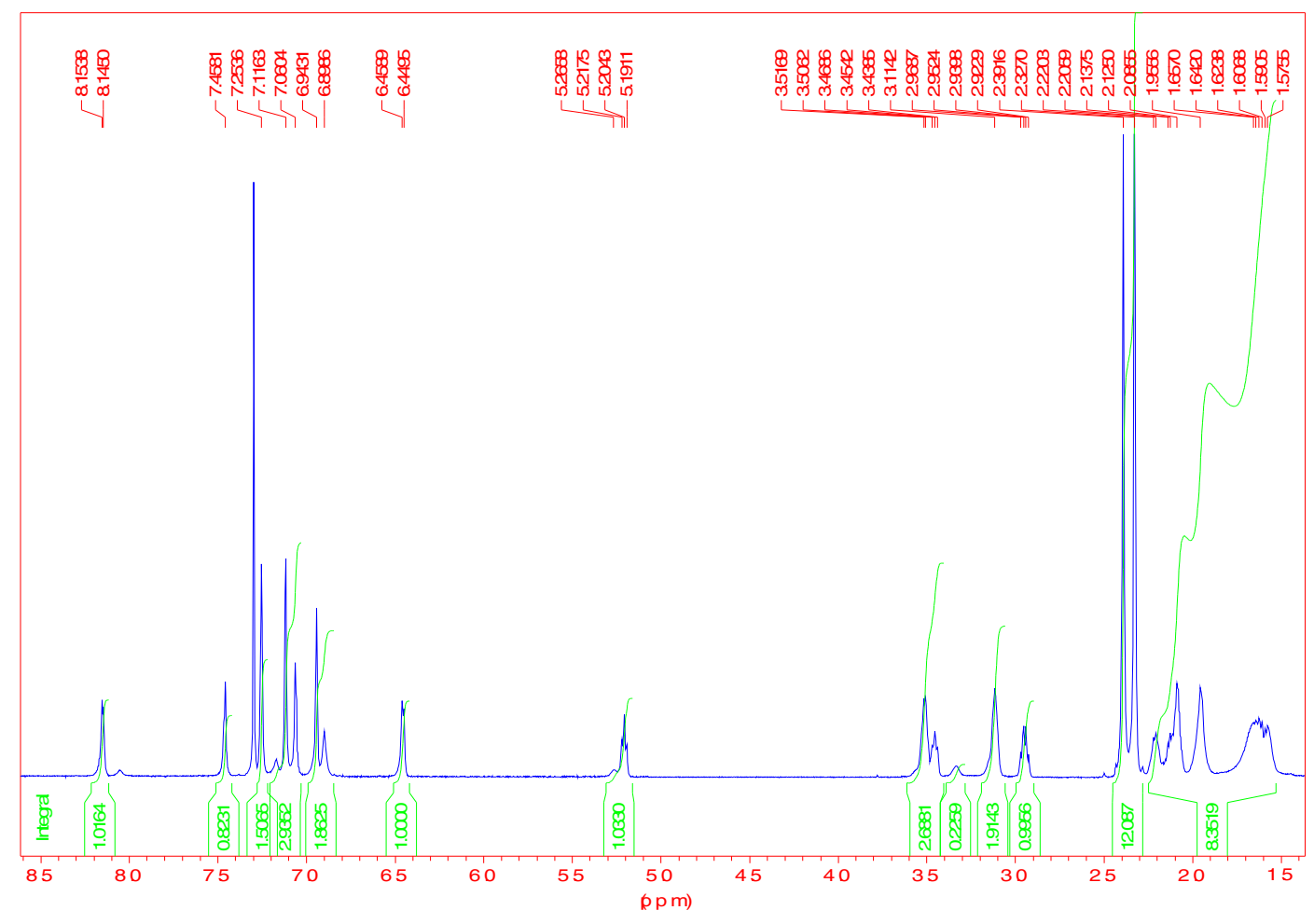

${ }^{13} \mathrm{C}$ NMR spectrum $\left(100 \mathrm{MHz}, \mathrm{CDCl}_{3}\right)$ of catalyst $\mathbf{1 d}$




${ }^{1} \mathrm{H}$ NMR spectrum $\left(400 \mathrm{MHz}, \mathrm{CDCl}_{3}\right.$ ) of catalyst 1e

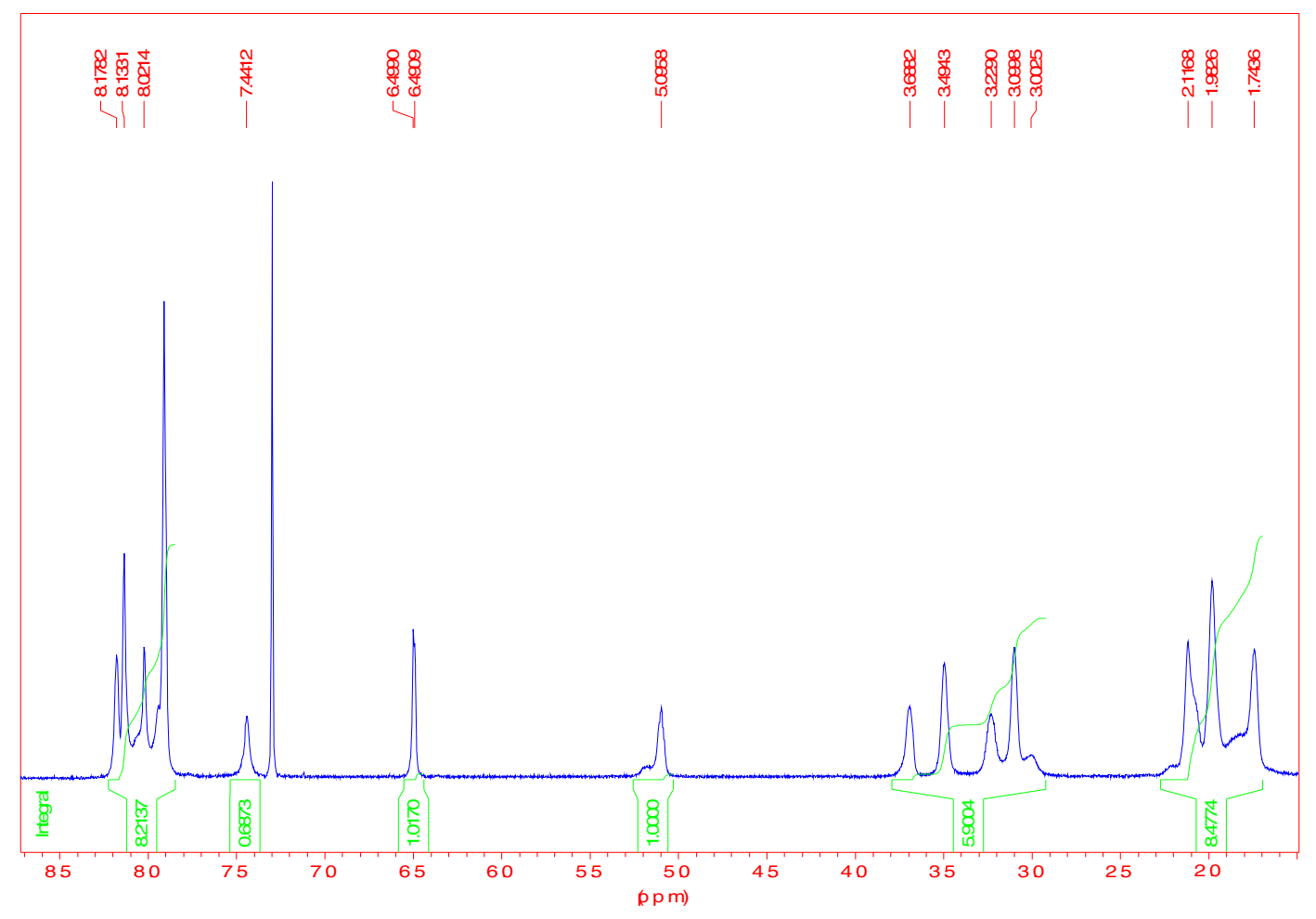

${ }^{13} \mathrm{C}$ NMR spectrum $\left(100 \mathrm{MHz}, \mathrm{CDCl}_{3}\right)$ of catalyst 1e




${ }^{1} \mathrm{H}$ NMR spectrum $\left(400 \mathrm{MHz}, \mathrm{CDCl}_{3}\right)$ of $\mathbf{9 a}$

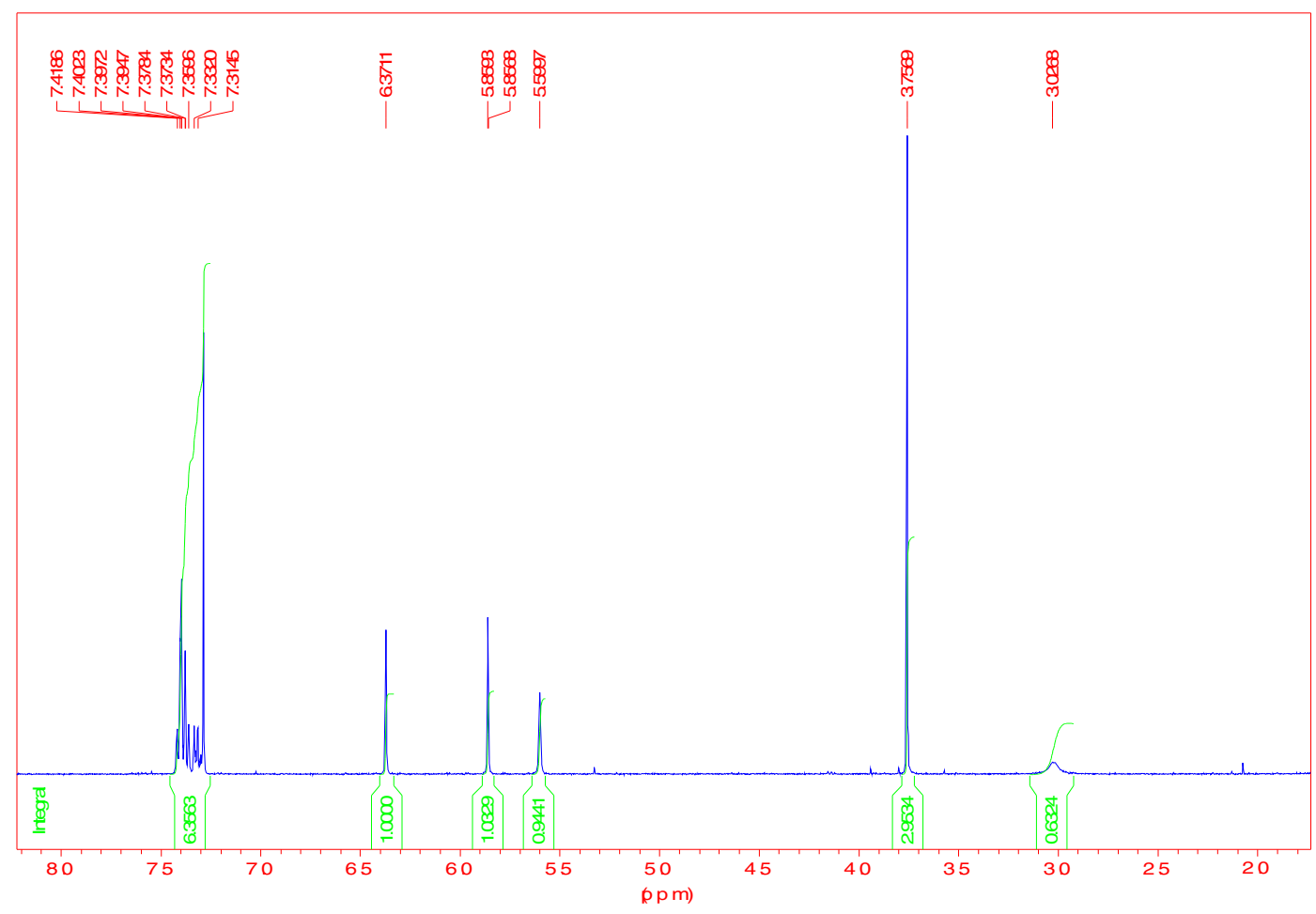

${ }^{1} \mathrm{H}$ NMR spectrum $\left(400 \mathrm{MHz}, \mathrm{CDCl}_{3}\right)$ of $\mathbf{1 0 a}$

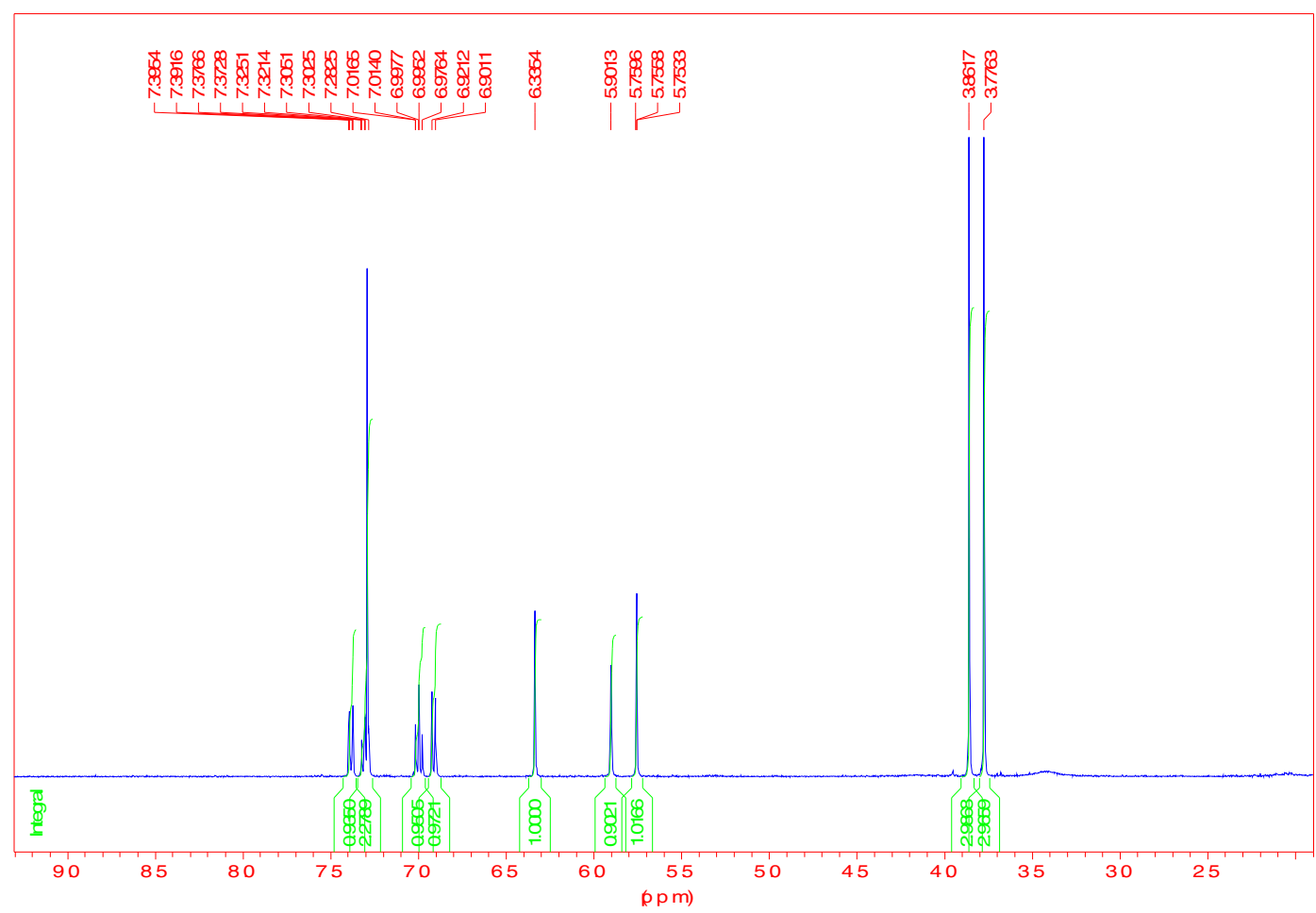


${ }^{1} \mathrm{H}$ NMR spectrum $\left(400 \mathrm{MHz}, \mathrm{CDCl}_{3}\right)$ of $\mathbf{1 1 a}$

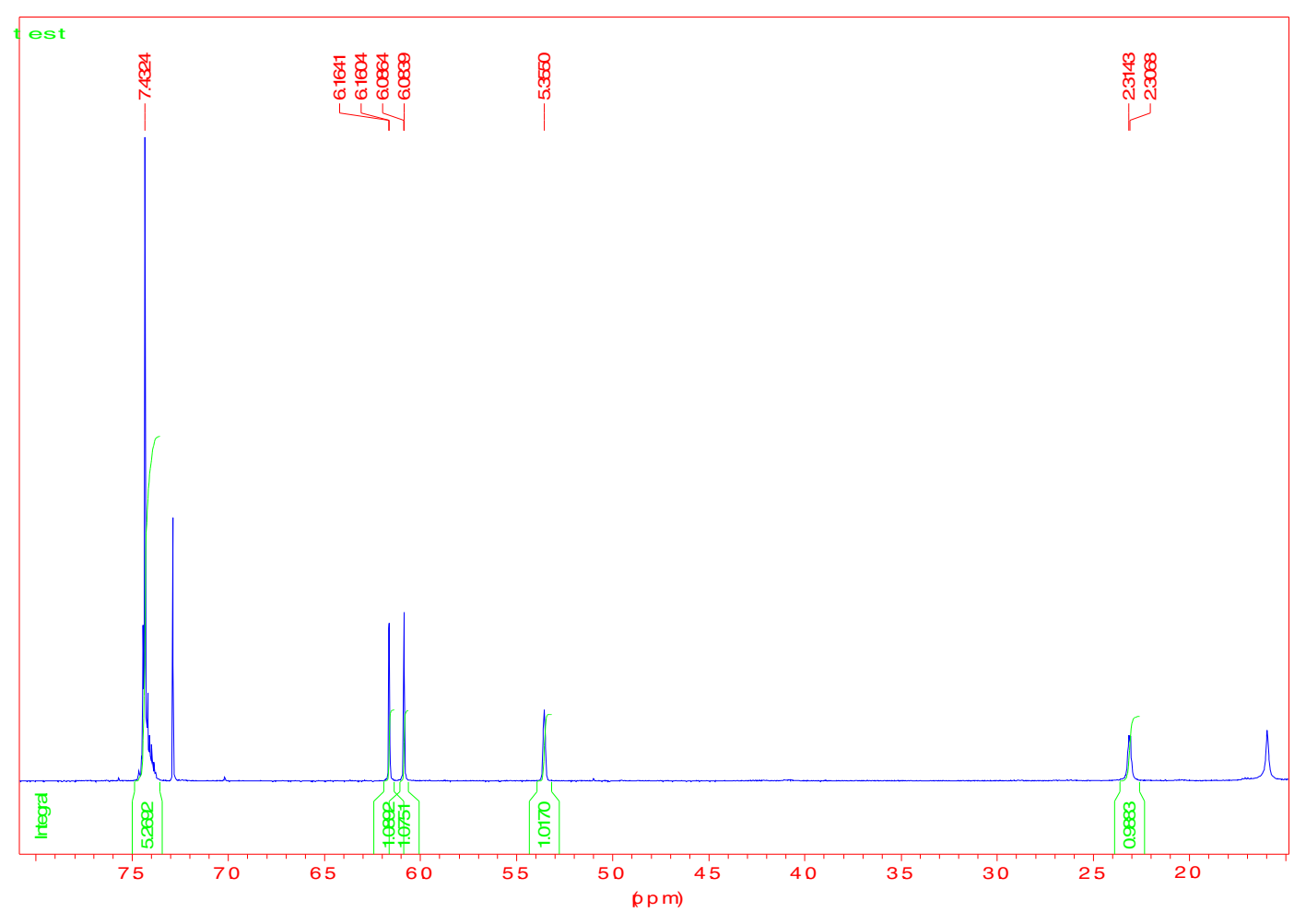

${ }^{1} \mathrm{H}$ NMR spectrum $\left(400 \mathrm{MHz}, \mathrm{CDCl}_{3}\right)$ of $\mathbf{1 2 a}$






\subsection{References}

1. Matsumoto, K.; Hatano, K.; Umezawa, N.; Higuchi, T. Synthesis 2004, 13, 2181.

2. Coleman, M. P.; Boyd, M. K. J. Org. Chem. 2002, 67, 7641.

3. Leazer Jr., J. L.; Cvetovich, R.; Tsay, F.-R.; Dolling, U.; Vickery, T.; Bachert, D. J. Org. Chem. 2003, 68, 3695.

4. Mathre, D. J.;Jones, T. K.; Xavier, L. C.; Blacklock, T. J.; Reamer, R. A.; Mohan, J. J.; Turner-Jones, E. T.; Hoogsteen, K.; Baum, M. W.; Grabowski, E. J. J. J. Org. Chem. 1991, 56, 751.

5. Marigo, M.; Wabnitz, T. C.; Fielenbach, D.; Jørgensen, K. A. Angew. Chem. Int. Ed. 2005, 44, 794 .

6. Shiina, I.; Yamai, Y.-S.; Shimazaki, T. J. Org. Chem. 2005, 70, 8103.

7 Drewes, S. E.; Emslie, N. D.; Field, J. S.; Khan, A. A.; Ramesar, N. Tetrahedron Asymm. 1992, 3, 255.

8. Priem, G.; Pelotier, B.; Macdonald, S. J. F.; Anson, M. S.; Campbell, I. B. J. Org. Chem. 2003, 68, 3844.

9. Birman, V. B.; Jiang, H.; Org. Lett. 2005, 7, 3445.

10. Kundig, E. P.; Xu, L. H.; Romanens, P.; Bernardinelli, G. Tetrahedron Lett. 1993, 34, 7049. 